\title{
Quantification of a Methadone Metabolite (EDDP) in Urine: Assessment of Compliance
}

\author{
Michael E.M. Larson, PhD and Thomas M. Richards, DO, FAAO
}

\begin{abstract}
Objective: To investigate the possibility of utilizing the ratio of the methadone metabolite, 2-ethylidene1,5-dimethyl-3,3-diphenylpyrrolidine (EDDP), to urine creatinine to develop a regression model that would predict drug adherence in patients prescribed methadone for either pain management or drug addiction.
\end{abstract}

Design: Retrospective study.

Setting: Marshfield Clinic-Lakeland Center, one of 4 I regional centers that make up Marshfield Clinic, a large, private, multi-specialty healthcare institution in central Wisconsin.

Participants: Patients receiving methadone treatment for substance abuse or chronic pain. Group I was an initial pilot group consisting of 7 patients who were followed for a 4-month period. Group 2 consisted of 33 patients who were followed over a 28-month period.

Methods: Age, gender, weight, height, methadone dosage, quantitative urine creatinine and EDDP levels, reported compliance/non-compliance, and relevant clinical cofactors were retrospectively abstracted from the patients' medical records. Log-log regression analyses were used to model EDDP and the EDDP/creatinine ratio from urine screening results as functions of methadone dose, and in the larger cohort (group 2), body size, gender and age. The coefficient of determination adjusted for the number of predictor terms $\left(\mathrm{R}_{\mathrm{adj}}{ }^{2}\right)$ was reported as a measure of model fit.

Results: For group I data, there was a significant positive relation $(P<0.00 \mathrm{I})$ but also substantial variability $\left(R_{\mathrm{adj}}{ }^{2}=0.49\right)$. Adjustment for creatinine through the EDDP/creatinine ratio provided a tighter relation $\left(\mathrm{R}_{\mathrm{adj}}{ }^{2}=0.95\right)$. Similarly, for group 2 data, there was a significant positive relation $(P=0.00 \mathrm{I})$ and substantial variability $\left(R_{\mathrm{adj}}{ }^{2}=0.53\right)$. Adjustment for creatinine through EDDP/creatinine ratios provided a substantially stronger relation $\left(R_{\text {adj }}{ }^{2}=0.73\right)$. Gender and age showed no evidence of association with the EDDP/creatinine ratio $(P=0.60$ and $P=0.5 \mathrm{I}$, respectively). Body size was significant in the model, both when measured by body surface area and by lean body weight, and improved the prediction when added to our model $\left(R_{\text {adj }}^{2}=0.80\right)$.

Conclusion: For the first time, urine analyses may be used to monitor methadone over- or under-use in a clinical setting, regardless of the state of patient hydration or the manipulation of a sample by addition of another substance, such as bleach, soap, or even methadone, which could render an appropriate sample inappropriate or an inappropriate sample appropriate. A similar approach may prove useful for other drug treatments, allowing for more accurate monitoring of commonly abused prescription medications.

Keywords: Chronic pain; Creatinine; Drug abuse; EDDP; Methadone; Urine specimen

Corresponding Author: Michael Larson, PhD, Department of Pain Management, Marshfield Clinic-Lakeland Center, 9601 Townline Road, PO Box 1390, Minocqua, WI 54548-I390, Tel: 7I5-358-I000, Fax: 7I5-358-I I56, Email: larson.michael@marshfieldclinic.org
Received: April 22, 2009 Revised: September 29, 2009 Accepted: October 7, 2009

doi: $10.3|2| / \mathrm{cmr} .2009 .859$
Grant Support:

Marshfield Clinic Research Foundation, Physician Research Funds

Patent:

U.S. Patent No. 7,585,680, M. Larson and T. Richards 
S patients in drug abuse programs or in patients with chronic pain who are on opioids for pain control. Although urine drug screening collection procedures attempt to ensure specimen integrity, some patients demonstrate considerable ingenuity in their efforts to defeat the testing process. ${ }^{1-4}$

Possible methods of avoiding detection of drug misuse include diversion, excessive water consumption, ingesting diuretics such as herbal teas, and substitution of someone else's urine. Individuals who divert pain medication for illegitimate resale often reserve a few pills to be taken before a physician visit so the medication will appear in their urine ensuring that the physician will extend the prescription, and therefore will allow continued diversion of the medication. Individuals also overuse medications, often gaining it from multiple sources. These individuals pass basic presence/ absence screening tests and continue to receive the medication. A recent retrospective study of a chronic pain population using urine screen monitoring suggested that $75 \%$ of patients were likely not taking their pain medication as prescribed. ${ }^{5}$

While laboratory assays are quantitative, current screening tests can only assure presence or absence of drug metabolites in the urine. The major problem facing urine testing is the large amount of variance in urine drug concentrations, mostly due to variations in hydration and urine output volume. In a recent study by the Clinical Pharmacology and Therapeutics Research Branch of the National Institute on Drug Abuse, this variability proved to be too great to permit estimation of methadone dose or missed doses; however, another group of researchers reported a clear benefit from the use of urine creatinine to adjust for concentration of urine methadone and its metabolite 2-ethylidene-1,5-dimethyl-3,3-diphenylpyrrolidine (EDDP) using a semiquantitative immunoassay and found that these values were lower on days after missed doses. ${ }^{6}$ Currently, no quantitative screening method is available that accurately identifies individuals who are noncompliant with the prescribed dosage regimen through overor under-use. Such a method would be of tremendous clinical benefit if it could be developed to allow statistical analysis of a single sample, thereby allowing a prescribing provider the ability to understand use of the medication as prescribed versus over- or under-use.

Oral creatine supplementation is widely used in athletes attempting to enhance their performance. This should not interfere with the proposed monitoring method. Several studies have demonstrated that neither short-, medium-, nor long-term oral creatine supplements have any significant effects on urine excretion rates for creatinine, urea or albumin in healthy individuals. 7,8 Of more concern is the fact that intraindividual variability in renal excretion of creatinine can be temporarily increased by meat consumption. ${ }^{9-11}$ This variability may account for $10 \%$ to $29 \%$ of between-day variation in calculated creatinine clearance for a given individual. ${ }^{12}$

The purpose of this study was to explore the possibility of using methadone metabolite/urine creatinine ratios to develop a regression model for predicting methadone adherence over a clinically significant range of prescribed dosages in patients on methadone therapy for chronic pain or opioid addiction. Methadone is prescribed under strict dosage regimens to patients in substance abuse programs, is closely monitored in chronic pain patients, and has a readily quantifiable urinary metabolite (EDDP). The direct methadone metabolite (methadone) was not assessed during this study, as it was not reported in these samples by our system's laboratory; Marshfield Laboratories only began reporting methadone levels quantitatively, along with EDDP levels, in February 2009. We sought to develop a model with predictive properties that could be used to compare predicted urine metabolite levels for a prescribed regimen against actual metabolite levels to determine non-adherence to a given regimen. We report our findings here.

\section{Methods}

Two sets of data were collected and analyzed. An initial pilot study (group 1) consisted of 39 total observations on seven unique patients. To supplement and validate the results from the group 1 data, an additional 102 observations were obtained in an additional 33 unique patients (group 2).

\section{Subjects}

Group 1. Patients receiving methadone treatment for substance abuse or chronic pain at Marshfield Clinic-Lakeland Center in Minocqua, Wisconsin over a 4-month period.

Group 2. Patients receiving methadone treatment for substance abuse or chronic pain at Marshfield Clinic-Lakeland Center in Minocqua, Wisconsin over a 28-month period.

For both groups, patient charts were reviewed and contained age, gender, height and weight data, a specified methadonedosing regimen, urine samples that included quantitative urine creatinine and EDDP levels, and documentation of the patients' reported compliance/non-compliance (eg, observed dose, missed appointment, ran out of medication secondary to transportation issues). Of the 141 total observations recorded, 20 observations ( 8 in group 1, and 12 in group 2) were determined to be invalid due to patient and/or clinical inquiries that led to reports of non-compliance with prescribed dosing. To prevent bias, laboratory results were not considered in determining compliance. Suspected non-compliant observations were not included in the statistical analyses.

Individual consent was obtained from qualifying patients according to Institutional Review Board protocols prior to chart abstraction. One patient did not agree to participate in this study resulting in exclusion of two observations from the 
analyses. For consenting patients, in addition to the information listed above, any clinical notes relevant to conditions expected to affect methadone metabolism were recorded.

\section{Urine Collection}

On a weekly basis, reasonably random urine screens were required of all methadone maintenance patients to assess for drugs of abuse. Chronic pain patients signed an opioid agreement requiring random urine screens for levels of (1) the prescribed drug, (2) other opioid medications, and (3) street or illicit drugs. No specific collection times, based on methadone dosing, were defined. This was not believed to affect metabolite detection outcomes. In future studies, urine collection times will be specified to eliminate this potential variable.

Urine was collected following the standard protocol for urine toxicity screening, such as for methadone and its metabolite EDDP. Other drugs were identified, but their impact on this study was not analyzed. Patients enrolled in this study were provided $30 \mathrm{~mL}$ specimen cups with temperature-sensitive strips (Lynn Peavey Co., Lenexa, KS). If urine temperature was appropriate for a "fresh" urine specimen $\left(90^{\circ}-100^{\circ} \mathrm{F}\right.$ per temperature strips), it was transferred to the laboratory for determination of urine creatinine level and a drug of abuse test that included EDDP quantitation via gas chromatography/ mass spectrometry (GC/MS) analysis.

\section{Creatinine Determinations}

Creatinine levels were determined employing the Jaffe reaction (DRI Creatinine-Detect, Microgenics Corp., Fremont, CA), whereby a red creatinine-picrate complex is formed. ${ }^{13}$ The rate of formation of the color product was measured using bichromatic pairing (absorbance at $505 \mathrm{~nm}$ minus nonspecific absorbance at $570 \mathrm{~nm}$ ). Samples were colorimetrically analyzed on a Hitachi 717 analyzer (Hitachi Chemical Diagnostics, Inc., Mountain View, CA). The reaction rate was used to construct a linear standard curve from which the concentration of creatinine was calculated.

\section{EDDP Determinations}

Aliquots of the urine specimens were first screened for drugs of abuse by routine immunoassay. Each presumptive positive specimen was subjected to confirmatory testing. In the case of methadone, EDDP was quantitatively measured by GC/MS with selected ion monitoring, following the method of Baugh et al ${ }^{14}$ with modifications as described below. Fifty microliters $(50 \mu \mathrm{L})$ of an internal standard (D3-EDDP, \# E-005, Cerilliant [formerly Radian], Round Rock, TX; $5 \mathrm{ng} / \mathrm{mL}$ in methanol) was added to $1 \mathrm{~mL}$ volumes of known EDDP controls (certified negative urine spiked with EDDP [\#01824, Alltech, Deerfield, IL] at 0, 120, 300, and $1500 \mathrm{ng} / \mathrm{mL}$ ) and urine test samples. All urine tests and known control samples were alkalized by adding $100 \mu \mathrm{L}$ of $25 \%$ (w/v) $\mathrm{NaOH}$ to $1 \mathrm{~mL}$ of sample to produce a $\mathrm{pH} 13$ to 14 . Because EDDP has no reactive functional groups, derivatization was not possible. EDDP and D3-EDDP were then liquid-liquid extracted by adding $3 \mathrm{~mL}$ of a chloroform:isopropanol (90:10 [v:v)]) mixture. After vortexing and centrifugation at $2800 \mathrm{rpm}$ for 5 min, the organic (bottom) layer was dried under nitrogen (N2) with gentle heat $\left(<37^{\circ} \mathrm{C}\right)$. Analytes in the tube were then reconstituted with $100 \mu \mathrm{L}$ of ethyl acetate and transferred to a $\mathrm{GC}$ vial for GC/MS analysis. A one-point calibration at the concentration of $300 \mathrm{ng} / \mathrm{mL}$ EDDP was used. The limit of quantification and the upper level of linearity was $40 \mathrm{ng} / \mathrm{mL}$ and $2000 \mathrm{ng} / \mathrm{mL}$, respectively.

Gas chromatography/mass spectrometry analysis was performed on the HP6890 GC connected to a HP5973 massselective detector (MSD) (Hewlett-Packard, Palo Alto, CA) and equipped with a $12 \mathrm{~m} \times 0.2 \mathrm{~mm}$ i.d., $0.3 \mu \mathrm{m}$ DB1 column (Agilent, Santa Clara, CA). Helium carrier gas was set to $0.5 \mathrm{~mL} / \mathrm{min}$ constant flow. Injector temperature was at $250^{\circ} \mathrm{C}$, and detector temperature was set to $280^{\circ} \mathrm{C}$. The oven temperature was programmed from $30^{\circ} \mathrm{C}$ (1-min hold) to $180^{\circ} \mathrm{C}$ at $40^{\circ} \mathrm{C} / \mathrm{min}$ then to $280^{\circ} \mathrm{C}$ at $20^{\circ} \mathrm{C} / \mathrm{min}$ (1-min hold). Samples were injected in splitless mode. The MSD data were collected using HP Chemstation G1710AA (version A.03.00) in selected ion monitoring (SIM) mode using the ions with the following mass-to-charge ratios (quantitation ions are underlined): D3-EDDP, 280, 265; EDDP, 277, 262, 276. Therefore, the fragment ions, EDDP and D3-EDDP, but not EMDP, were monitored to determine methadone in the urine samples. The internal standard made a small contribution to the analyte ion chromatograms, especially to the 262 ion, limiting the sensitivity of the assay to a limit of detection of approximately $10 \mathrm{ng} / \mathrm{mL}$. All samples were above the limit of detection and were appropriately quantified.

\section{Urine Creatinine Correction}

EDDP/creatinine ratios were calculated by dividing the EDDP level $(\mathrm{ng} / \mathrm{mL})$ by the urine creatinine level $(\mathrm{mg} / \mathrm{dL})$.

\section{Body Size}

Body surface area (BSA) was calculated according to the Mosteller formula: ${ }^{15}$

$$
\text { BSA }\left(\mathrm{m}^{2}\right)=([\text { height }(\mathrm{cm}) \times \text { weight }(\mathrm{kg})] / 3600)^{1 / 2}
$$

Lean body weight (LBW) was calculated as follows: ${ }^{16}$ LBW $($ men $)=(1.10 \times$ weight $(\mathrm{kg}))-128 \times\left(\right.$ weight $\left.^{2} /[100 \times \text { height }(\mathrm{m})]^{2}\right)$ LBW $($ women $)=(1.07 \times$ weight $(\mathrm{kg}))-148 \times\left(\right.$ weight $\left.^{2} /[100 \times \text { height }(\mathrm{m})]^{2}\right)$

\section{Data Analysis}

Regression analyses were used to model EDDP and the EDDP/creatinine ratio from valid urine screening results as functions of methadone dose, and in the larger cohort (group 2), body size, gender and age. All analyses were conducted in a repeated measures (mixed linear model) ${ }^{17}$ framework to allow for correlation among multiple observations from the same patient. Models were fit after logarithmic transformation of the laboratory and dose measurements to adjust for skewness in the distribution and improve the linearity of the relations. Backward selection was used to remove non-significant terms from the models when 
Table 1. Patient characteristics are similar in both groups.

\begin{tabular}{lcc}
\hline & Group 1 & Group 2 \\
\hline Number of patients & 7 & 33 \\
Male & $3(43 \%)$ & $21(64 \%)$ \\
Female & $4(57 \%)$ & $12(36 \%)$ \\
Age range & $25-51$ & $20-56$ \\
Observations & 39 & 102 \\
Compliant & $31(79 \%)$ & $90(88 \%)$ \\
Non-compliant & $8(21 \%)$ & $12(12 \%)$ \\
LBW range (kg) & $45-69$ & $38-83$ \\
BSA range & $1.65-2.21$ & $1.54-2.87$ \\
\hline BSA, body surface area; LBW, lean body weight & & \\
\hline
\end{tabular}

considering additional factors (such as an indicator for male gender, age and body size as continuous predictors), and the coefficient of determination adjusted for the number of predictor terms $\left(\mathrm{R}_{\mathrm{adj}}{ }^{2}\right)$ was reported as a measure of model fit. Plots of the data and analyses of the residuals and predicted values from the regression models were used to ensure that the final models adequately represented the observed data. ${ }^{18}$ Observations judged "non-compliant" were not included in the development of the models, but are included in plots to illustrate deviations from valid results predicted by the models. The predictive ability of the final model is illustrated with sensitivity ( $\%$ of non-compliant observations correctly identified) and specificity (\% of compliant observations correctly identified), where this classification is based on the studentized (ie, normalized) residuals from the model. These studentized residuals $\left(\mathrm{r}_{\mathrm{s}}\right)$ might be used, as with other clinical Z-scores, to flag unusual values, in this case as potentially reflecting non-compliance. Results in this report were deemed statistically significant at the 5\% level $(P<0.05)$. Other substance metabolites (eg, oxycodone, morphine) may have been present in the urine screens; however, for the purpose of this retrospective study, they were not included in this data set and were not analyzed in any way.

\section{Results}

Patient Characteristics

Table 1 describes the patients and their urine screening observations. The seven patients in group 1 ranged in age from 25 to 51 years, and $3(43 \%)$ were male. The 33 patients in group 2 ranged in age from 20 to 56, and 21 (64\%) were male. Of the 40 total patients, 3 were receiving methadone therapy for substance abuse, with the remaining 37 receiving the therapy for chronic pain management. All patients were considered to have achieved "steady state" and were taking medication on a consistent dosing schedule at the time of the assessments; the only exceptions were when non-compliance with the prescribed dosing regimen had been documented.

Predictive Modeling - Group 1

Figure $1 \mathrm{~A}$ indicates a significant positive relation $(P<0.001)$

\section{A}

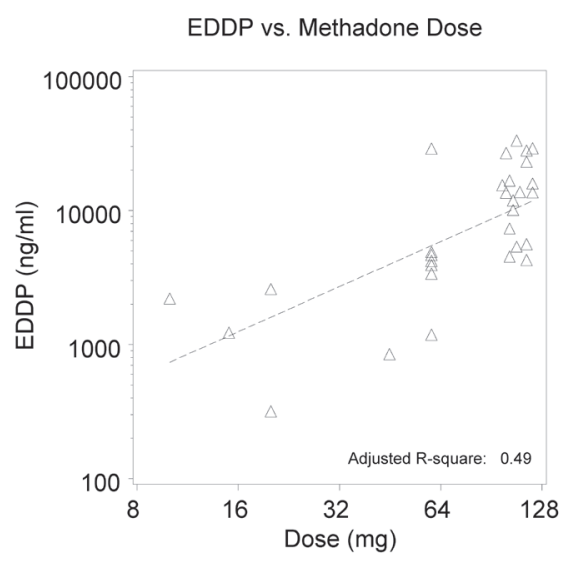

B

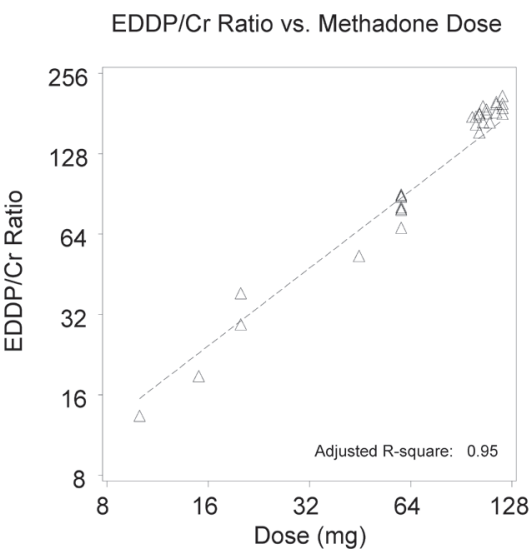

C

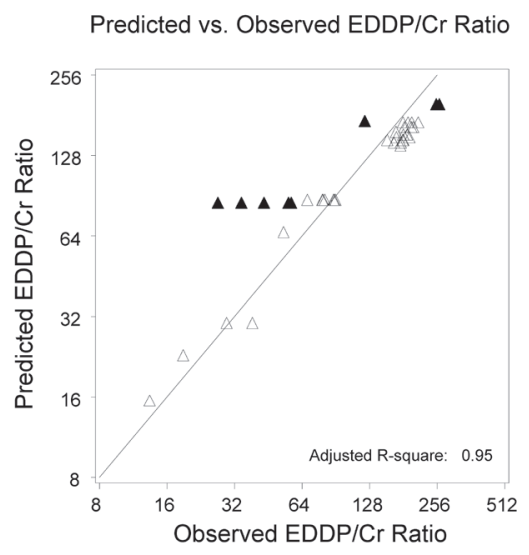

Figure 1. (A) Group 1 uncorrected urine EDDP levels for confirmed daily methadone doses. The fitted line from the linear regression model (log scale) indicates a positive relation. (B) Group 1 laboratory EDDP/creatinine ratios from urine of confirmed daily methadone doses. The fitted line from the linear regression model (log scale) reduces variability of the positive relation. (C) Non-compliant observations in group 1 deviate from the predicted versus observed EDDP/creatinine ratios (log scale) ( $\boldsymbol{\Delta}=$ non-compliant dosing, $\Delta=$ methadone daily doses). 
A

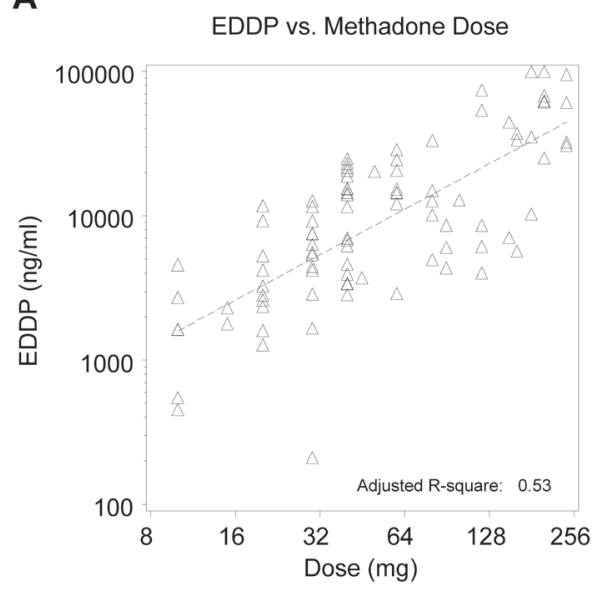

B

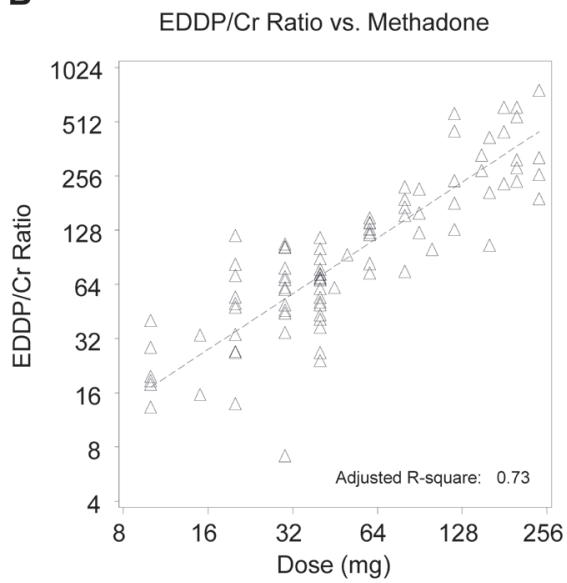

C

Predicted vs. Observed EDDP/Cr Ratio

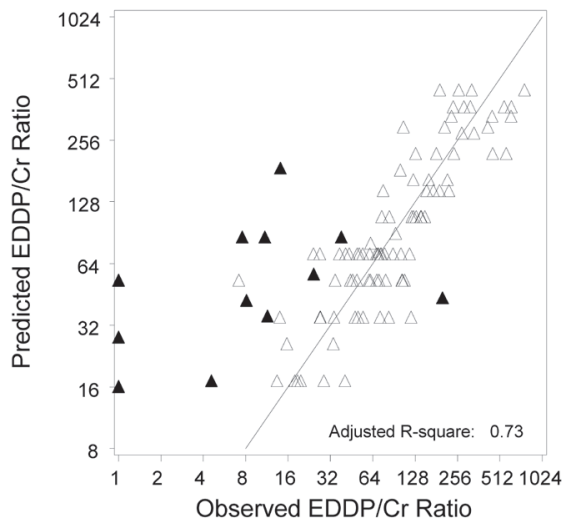

Figure 2. (A) Group 2 uncorrected urine EDDP levels of confirmed daily methadone doses are also positively related, but variability remains high. (B) Adjustment using urine EDDP/creatinine ratios for confirmed daily methadone doses of group 2 indicates a stronger correlation and is shown by the fitted line from the linear regression model (log scale). (C) Prediction of urine EDDP/creatinine ratios versus observed group $2 \mathrm{EDDP} / \mathrm{creatinine} \mathrm{ratios} \mathrm{demonstrates} \mathrm{divergence} \mathrm{from} \mathrm{the} \mathrm{line} \mathrm{of} \mathrm{identity}$ (log scale) for non-compliant observations ( $\boldsymbol{\Delta}=$ non-compliant dosing, $\Delta=$ methadone daily doses).

of the valid EDDP results by dose from group 1. However, there was also substantial variability $\left(\mathrm{R}_{\mathrm{adj}}{ }^{2}=0.49\right)$. As shown in figure $1 \mathrm{~B}$, adjustment for creatinine using $\mathrm{EDDP} /$ creatinine ratios provided a much tighter relation $\left(\mathrm{R}_{\mathrm{adj}}{ }^{2}=0.95\right)$. Figure 1C illustrates the fit of the same model by plotting EDDP/creatinine as predicted by the model versus the observed value, and includes the identity line representing perfect prediction by the model. The confirmed non-compliant dose values, as expected, deviate substantially from the predicted values.

\section{Predictive Modeling - Group 2}

In order to validate the results above, the analyses were repeated in a larger, independent group of patients. The valid EDDP results by dose for group 2 are shown in figure 2A. Once again, there was a significant positive relation $(P<0.001)$ but also substantial variability $\left(\mathrm{R}_{\mathrm{adj}}{ }^{2}=0.53\right)$. As in the original group of patients, adjustment for creatinine through the EDDP/creatinine ratio provided a substantially stronger relation $\left(\mathrm{R}_{\mathrm{adj}}{ }^{2}=0.73\right.$ ) (figure $\left.2 \mathrm{~B}\right)$. The fit of the same model by plotting EDDP/creatinine as predicted by the model versus the observed value for group 2 is shown in figure $2 \mathrm{C}$ and includes the identity line representing perfect prediction by the model. A plot of EDDP/creatinine ratios predicted by the model versus the observed EDDP/creatinine values again indicates considerable deviation for confirmed non-compliant dosing (figure 2C).

\section{Predictive Modeling - Combined Groups}

In additional analyses, all patients were combined in order to consider whether patient characteristics may also relate to the EDDP/creatinine ratio. Through multiple regression modeling, we evaluated gender, age, and body size as covariates. Neither gender nor age showed evidence of association with the $\mathrm{EDDP} /$ creatinine ratio $(P=0.38$ and $P=0.35$, respectively).
Body size, however, was significant in the model, both when measured by BSA $(P=0.003)$ and when measured by LBW $(P<0.001)$. Adding LBW to our model improved the prediction $\left(\mathrm{R}_{\mathrm{adj}}{ }^{2}=0.80\right)$ as shown in figure 3 , with the prediction equation as follows:

$$
\begin{aligned}
& \log _{10}(\mathrm{EDDP} / \text { creatinine })=0.68-0.008 * \mathrm{LBW}+1.03 * \log _{10} \\
& (\text { methadone dose in } \mathrm{mg}) .
\end{aligned}
$$

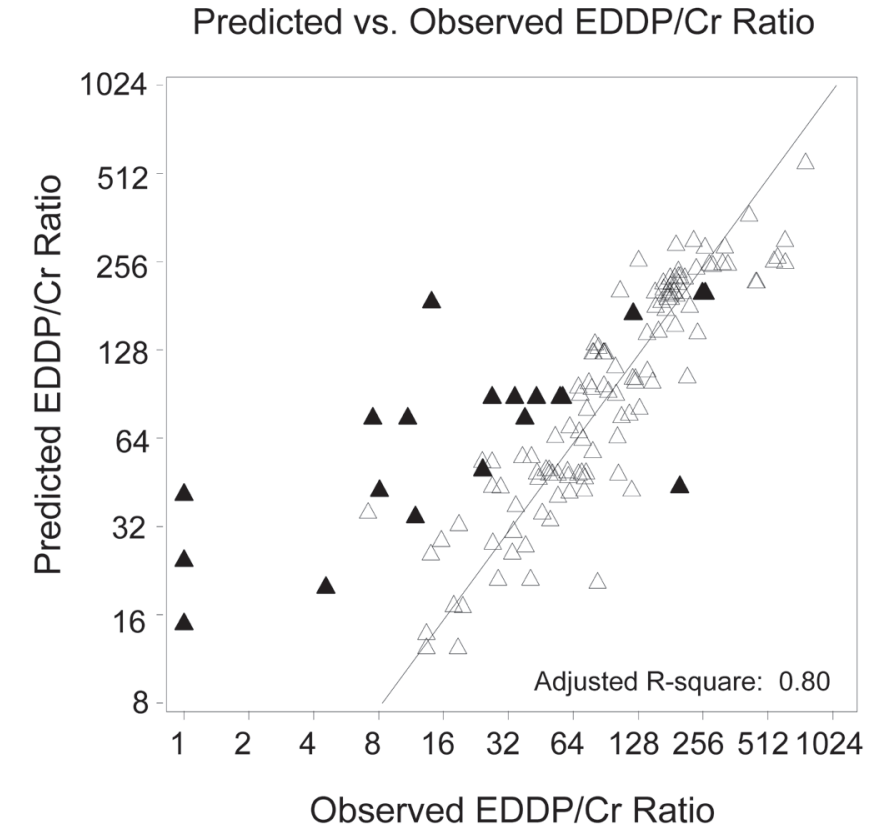

Figure 3. Variability of the line of identity (log scale) in the model decreases when EDDP/creatinine ratios are adjusted for LBW, using combined data from group 1 and 2 ( $\boldsymbol{\Delta}=$ non-compliant dosing, $\Delta=$ methadone daily doses). 
Applying this prediction equation to the 20 non-compliant observations and evaluating residuals finds $75 \%$ exceeding $\left|\mathrm{r}_{\mathrm{s}}\right|>2$ and $60 \%$ exceeding $\left|\mathrm{r}_{\mathrm{s}}\right|>3$, as compared with only $3.3 \%$ and $1.7 \%$ of 121 compliant obervations, respectively. Thus, a decision rule of $\left|\mathrm{r}_{\mathrm{s}}\right|>2$ shows estimated sensitivity/specificity of $75 \% / 96.7 \%$, while $\left|r_{s}\right|>3$ shows estimated sensitivity/ specificity of $60 \% / 98.3 \%$.

\section{Discussion}

A relationship between urinary $\mathrm{EDDP} /$ creatinine ratios was found to predict the consumed dosage of methadone across the clinically significant range used in drug abuse and chronic pain patients. This confirms the previous results using EDDP with creatinine correction and further was able to confirm noncompliance in the form of over- or under-use. ${ }^{6}$ Correction for creatinine removed a substantial proportion of the variability associated with urine testing results. Documented aberrant methadone use in the methadone maintenance patients was readily apparent. If this model is further validated, it could substantially benefit methadone maintenance and/or chronic pain programs that use methadone, by improving the clinician's ability to objectively monitor patient adherence to dosage regimens. Although this was not a clinical model, it could readily be developed with additional research and would allow clinicians to have better information about how their patient is using the prescribed medication, leading to better clinical decision-making. Future research may also look at the additional benefit of utilizing EDDP along with methadone level to improve confidence intervals and the ability to identify appropriate versus inappropriate therapy use.

It is important for clinicians to be aware of any patient's inappropriate use of prescribed, over-the-counter, and/or illicit drugs to properly manage care. Although use of external sources of information may be helpful (such as interviews with spouses, review of medical records, or input from prescription monitoring programs), testing of biological samples, especially urine, has the greatest potential for monitoring true compliance. ${ }^{19}$

Hawks $^{20}$ first suggested using creatinine normalization to correct for variations in metabolite urine tests. Creatinine is a metabolite of creatine and is an end-product of muscle metabolism excreted in the urine. Creatinine formation and excretion are directly proportional to total muscle mass and roughly proportional to body weight. Creatinine is excreted in relatively constant amounts of 1.0 to $2.5 \mathrm{~g}$ /day regardless of urinary volume, ${ }^{9,10}$ and normalization of the excretion of a drug to the creatinine concentration may reduce the variability of analyte measurement attributed to urine dilution. Total 24-hour creatinine determinations have been utilized for checks on 24-hour urine collection adherence. Also, in forensic science, drug metabolite to creatinine ratios are utilized for reducing false negative results caused by urine specimen dilution. Creatinine ratios have not, however, been applied to assessments of whether patients are adhering to prescribed drug treatment regimens.
Normalizing urine drug concentrations to urine creatinine values has been attempted for drugs such as marijuana, 21-24 amphetamine, ${ }^{25}$ cocaine, ${ }^{26}$ nicotine, ${ }^{27}$ and buprenorphine. ${ }^{28}$ Most of these applications, however, have been designed with the specific aim of avoiding false negative results in drug screening programs due to very dilute urine specimens. ${ }^{22,29,30}$ Few, however, have attempted to employ creatinine adjustments in the clinical setting. Manno et al ${ }^{23}$ reported that the Syva EMIT-d.a.u. urine cannabinoid assay (Dade Behring, Palo Alto, CA) could be successfully used for detecting marijuana use patterns in a urine surveillance program if creatinine corrections were applied. They reported that a delta-9-tetrahydrocannabinol-9-carboxylic acid (THCA)/ creatinine ratio should decrease over time when there is no new use and recommended that when comparing results, the THCA/creatinine ratio should decrease by $50 \%$ every 2 to 10 days, depending on the individual. More recently, studies have confirmed the usefulness of the THCA/creatinine ratio under controlled-dosing conditions with marijuana smokers. ${ }^{31,32}$ Sequential creatinine normalized urine drug concentrations could predict whether marijuana exposure had re-occurred, or if the presence of THCA in urine was due to continued clearance from the body in $85 \%$ of the cases. Additionally, reports prove that ratios in a light or infrequent user will decrease faster than those in a heavy or frequent user. Cone et $\mathrm{al}^{33}$ reported on normalization of cocaine urine testing results to urine creatinine. Quantitative benzoylecgonine (BE) to urine creatinine levels allowed for the differentiation of positive BE levels arising from washout and from positive BE levels resulting from new cocaine use. ${ }^{26}$ Preston et al ${ }^{6}$ reported the benefit of this method for correcting to creatinine, for methadone and its main metabolite EDDP, when they are determined by semiquantitative CEDIA DAU immunoassays. Our study suggests that the same approach may be useful in monitoring methadone adherence in clinically relevant situations using GC/MS.

Limitations remain, however. This was a retrospective analysis. The patients included in this study all had presumptive positive urine screens utilizing immunoassays that were verified by GC/MS-selected ion monitoring. Individuals who may have been false negative on immunoassay were not included, so it is not known how the model would have predicted dosage adherence in such patients. Also, other substances may have been present in the urine, and it is unknown the impact of those substances on the current findings, although prior research suggests these other metabolites would not impact the findings in a significant manner. ${ }^{6}$ Future research may need to continue to investigate the interaction between various metabolites on the confidence intervals. Further analyses have been suggested to determine how the methadone metabolite, along with EDDP, in combination with adjustment to urine creatinine may improve the confidence intervals. Additionally, other factors such as urine $\mathrm{pH}$ or specific gravity may allow improved confidence intervals and associated predictive value of urine testing. These parameters were not considered in this study, but may 
be worthy of consideration in future studies as they have been found to provide interpretative benefit. ${ }^{5}$ We present our predictive model for illustration, and realize it will require further development and validation prior to any clinical application.

\section{Conclusion}

Our data were sufficient to yield a highly significant regression that permitted the demonstration of known outliers. If such a model is established and supported with additional data from a larger normative sample, it may result in improved monitoring of medication usage and eventually lead to identification of misuse, diversion tactics, or neglectful non-adherence. Patients treated with opioids for the management of chronic pain have been documented to under-report their use of medications, especially for the opioid class of medications. ${ }^{19,34-38}$ A higher level of verifiable control may be achievable. Confident medication management by practitioners may be enhanced by objective evidence that the patient appears to be taking the medication correctly, especially when the lab results are reported in a format that clinicians are comfortable seeing (ie, confidence intervals within two standard deviations from the mean). The long half-life of methadone makes this an ideal candidate for this type of objective testing, but this may also apply to other medications that have a high tendency for misuse.

If further validation of this model is achieved, potentially with new factors that would allow even tighter confidence intervals, not only for methadone, but also for other opioid medications (eg, oxycodone or morphine products) or psychostimulant medications, many of which are abused, misused or diverted, a dramatic improvement in a physicians ability to manage medications appropriately could be attained. This could lead to physicians identifying medication diverters or abusers more quickly, thus leading to a dramatic reduction in risk to patients, abusers and the community. The current urine screen model, which is clearly inadequate, would be much improved and the overall decision-making by physicians in the context of medication could be substantially advanced.

\section{Acknowledgments}

The authors thank the Marshfield Clinic Research Foundation for providing assistance in the preparation of this manuscript through the services of the Office of Scientific Writing and Publication and also thank the Clinical Research Center for assistance in navigating the research process. We also thank Richard Berg for his assistance with the statistical analysis of the data; the Division of Laboratory Medicine, Toxicology Services at Marshfield Labs for performing the creatinine and EDDP determinations; and the Marshfield Clinic Minocqua Center, Department of Pain Management for assisting in the clinical activities that allowed the completion of this research.

\section{References}

1. Barbanel CS, Winkelman JW, Fischer GA, King AJ. Confirmation of the Department of Transportation criteria for a substituted urine specimen. J Occup Environ Med 2002;44:407-416.

2. Kapur B, Hershkop S, Koren G, Gaughan V. Urine fingerprinting: detection of sample tampering in an opiate dependency program. Ther Drug Monit 1999;21:243-250.

3. Kintz P, Tracqui A, Mangin P, Edel Y. Sweat testing in opioid users with a sweat patch. J Anal Toxicol 1996;20:393-397.

4. Preston KL, Huestis MA, Wong CJ, Umbricht A, Goldberger BA, Cone EJ. Monitoring cocaine use in substance-abusetreatment patients by sweat and urine testing. J Anal Toxicol 1999;23:313-322.

5. Couto JE, Romney MC, Leider HL, Sharma S, Goldfarb NI. High rates of inappropriate drug use in the chronic pain population. Popul Health Manag 2009;12:185-190.

6. Preston KL, Epstein DH, Davoudzadeh D, Huestis MA. Methadone and metabolite urine concentrations in patients maintained on methadone. J Anal Toxicol 2003;27:332-341.

7. Poortmans JR, Auquier H, Renaut V, Durussel A, Saugy M, Brisson GR. Effect of short-term creatine supplementation on renal responses in men. Eur J Appl Physiol Occup Physiol 1997;76:566-567.

8. Poortmans JR, Francaux M. Long-term oral creatine supplementation does not impair renal function in healthy athletes. Med Sci Sports Exerc 1999;31:1108-1110.

9. Alessio L, Berlin A, Dell'Orto A, Toffoletto F, Ghezzi I. Reliability of urinary creatinine as a parameter used to adjust values of urinary biological indicators. Int Arch Occup Environ Health 1985;55:99-106.

10. Bingham SA, Cummings JH. The use of creatinine output as a check on the completeness of 24-hour urine collections. Hum Nurt Clin Nutr 1985;39:343-353.

11. Tietz N. Textbook of clinical chemistry. Philadelphia, PA: WB Saunders Co; 1986. 1279-1290.

12. Narayanan S, Appleton HD. Creatinine: a review. Clin Chem 1980;26:1119-1126.

13. Butler AR. The Jaffé reaction. Identification of the coloured species. Clin Chim Acta 1975;59:227-232.

14. Baugh LD, Liu RH, Walia AS. Simultaneous gas chromatography/mass spectrometry assay of methadone and 2-ethyl-1,5-dimethyl-3,3-diphenylpyrrolidine (EDDP) in urine. J Forensic Sci 1991;36:548-555.

15. Mosteller RD. Simplified calculation of body-surface area. N Engl J Med 1987;317:1098.

16. halls.md. Formulas for calculating body surface area (BSA). Available at: http://www.halls.md/body-surface-area/refs.htm. Accessed July 2003.

17. Littell RC, Milliken GA, Stroup WW, Wolfinger RD. SAS System for Mixed Models. Cary, North Carolina, SAS Institute Inc., 1996.

18. Neter J, Wasserman W. Applied Linear Statistical Models. Homewood, Illinois, Richard D. Irwin, 1974.

19. Katz N, Fanciullo GJ. Role of urine toxicology testing in the management of chronic opioid therapy. Clin J Pain 2002; 18(4 suppl):S76-S82.

20. Hawks RL. Developments in cannabinoid analyses of body fluids: implications for forensic applications. In: Agurell S, Dewey W, Willette R, eds. The cannabinoids: chemical, pharmacologic and therapeutic aspects. Rockville, Maryland: Academic Press; 1983:1-12.

21. Bell R, Taylor EH, Ackerman B, Pappas AA. Interpretation of urine quantitative 11-nor-delta-9 tetrahydrocannabinol-9carboxylic acid to determine abstinence from marijuana smoking. J Toxicol Clin Toxicol 1989;27:109-115. 
22. Lafolie P, Beck O, Blennow G, Boréus L, Borg S, Elwin CE, Karlsson L, Odelius G, Hjemdahl P. Importance of creatinine analyses of urine when screening for abused drugs. Clin Chem 1991;37:1927-1931.

23. Manno J, Ferslew KE, Manno BR. Urine excretion patterns of cannabinoids and the clinical application of the EMIT-dau cannabinoid urine assay for substance abuse treatment. In: Agurell A, Dewey WE, Willet RE, eds. The cannabinoids: chemical pharmacologica and therapeutic aspects. New York: Academic Press; 1984:281.

24. Painter PC, Evans JH, Greenwood JD, Fain WW. Urine cannabinoids monitoring. Diag Clin Testing 1989;27:29-33.

25. Jones AW, Karlsson L. Relation between blood- and urineamphetamine concentrations in impaired drivers as influenced by urinary $\mathrm{pH}$ and creatinine. Hum Exp Toxicol 2005;24:615-622.

26. Weiss RD, Gawin FH. Protracted elimination of cocaine metabolites in long-term high-dose cocaine abusers. Am J Med 1988;85:879-880.

27. Thompson SG, Barlow RD, Wald NJ, Van Vunakis H. How should urinary cotinine concentrations be adjusted for urinary creatinine concentration? Clin Chim Acta 1990;187:289-295.

28. Watson ID. Analysis of commonly abused drugs in urine at selected threshold (cutoff) concentrations. Clin Chem 1992;38:441.

29. Cone EJ, Lange R, Darwin WD. In vivo adulteration: excess fluid ingestion causes false-negative marijuana and cocaine urine test results. J Anal Toxicol 1998;22:460-473.

30. Needleman SB, Porvaznik M, Ander D. Creatinine analysis in single collection urine specimens. J Forensic Sci 1992;37:1125-1133.

31. Fraser AD, Worth D. Urinary excretion profiles of 11-nor-9carboxy-Delta9-tetrahydrocannabinol: a Delta9-THC-COOH to creatinine ratio study \#2. Forensic Sci Int 2003;133:26-31.

32. Huestis M, Cone E. Differentiating new marijuana use from residual drug excretion in occasional marijuana users. J Anal Toxicol 1998;22:445-454.

33. Cone EJ, Caplan YH, Moser F, Robert T, Shelby MK, Black DL. Normalization of urinary drug concentrations with specific gravity and creatinine. J Anal Toxicol 2009;33:1-7.

34. Berndt S, Maier C, Schütz HW. Polymedication and medication compliance in patients with chronic non-malignant pain. Pain 1993;52:331-339.

35. Chabal C, Erjavec MK, Jacobson L, Mariano A, Chaney E. Prescription opiate abuse in chronic pain patients: clinical criteria, incidence and predictors. Clin J Pain 1997; 13:150-155.

36. Fishman SM, Bandman TB, Edwards A, Borsook D. The opioid contract in the management of chronic pain. J Pain Symptom Manage 1999;18:27-37.

37. Ready LB, Sarkis E, Turner JA. Self-reported vs. actual use of medications in chronic pain patients. Pain 1982;12:285-294.

38. Sees KL, Clark HW. Opioid use in the treatment of chronic pain: assessment of addiction. J Pain Symptom Manage $1993 ; 8: 257-264$.

\section{Author Affiliations}

Michael E.M. Larson, PhD*; and

Thomas M. Richards, DO, FAAO';

Department of Pain Management*, and

Department of Neuromusculoskeletal Medicinet

Marshfield Clinic Minocqua Center

Minocqua, Wisconsin, USA 